\title{
The impacts of faecal subsampling on microbial compositional profiling
}

\author{
Amanda J. Cox ${ }^{1,2^{*}} \mathbb{0}$, Lily Hughes ${ }^{1}$, Tiffanie M. Nelson ${ }^{2,3}$, Kyle M. Hatton-Jones ${ }^{1}$, Rebecca Ramsey ${ }^{1}$, \\ Allan W. Cripps ${ }^{2,4}$ and Nicholas P. West ${ }^{1,2}$
}

\begin{abstract}
Objective: Despite the move to at-home, small-volume collection kits to facilitate large population-based studies of faecal microbial compositional profiling, there remains limited reporting on potential impacts of faecal subsampling approaches on compositional profiles. This study aimed to compare the microbial composition from faecal subsamples $(<5 \mathrm{~g})$ collected from the beginning and end of a single bowel movement in ten otherwise healthy adults ( 6 female, 4 male; age: 24-55 years). Microbial composition was determined by V3-V4 16s rRNA sequencing and compared between subsamples.

Results: There were no significant differences in OTU count $(p=0.32)$ or Shannon diversity index $(p=0.29)$ between the subsamples. Comparison of relative abundance for identified taxa revealed very few differences between subsamples. At the lower levels of taxonomic classification differences in abundance of the Bacillales $(p=0.02)$ and the Eubacteriaceae family $(p=0.03)$, and the Eubacterium genera $(p=0.03)$ were noted. The observation of consistent microbial compositional profiles between faecal subsamples from the beginning and end of a single bowel movement is an important outcome for study designs employing this approach to faecal sample collection. These findings provide assurance that use of a faecal subsample for microbial composition profiling is generally representative of the gut luminal contents more broadly.
\end{abstract}

Keywords: Gut microbiota, Faecal samples, Sub-sampling, 16s rRNA

\section{Introduction}

With exponential growth in the numbers of studies exploring the contribution of the gut microbiota to health and disease over the past decade, aligning sample collection approaches and analytical methods is crucial if the growing collection of outcomes are to be compared. Indeed, large numbers of studies have now assessed the impacts that differences in sample collection techniques (e.g. sample cups, swab-based collection kits), storage conditions (e.g. room temperature, immediate freezing, with/without stabilizing buffer), processing methods (e.g.

\footnotetext{
*Correspondence: a.cox@griffith.edu.au

1 School of Medical Science, Griffith Health Centre (G40), Griffith

University, Parklands Drive, Southport, QLD 4215, Australia

Full list of author information is available at the end of the article
}

with/without chemical and mechanical lysis), selection of 16s rRNA hypervariable regions for library preparation and sequencing, and data analysis pipelines (e.g. use of different reference databases) can have on microbial composition (see reviews, [1, 2]). The impact of these methodological factors on diversity metrics [3], detection of gram-positive bacteria [4], detection of anaerobic bacteria [5], and estimation of low-abundance/rare taxa [6] have all been acknowledged.

To support the recruitment of large, often geographically distant cohorts, earlier sample collection methods suggesting homogenization of the entire bowel movement and subsampling of the homogenate for subsequent compositional profiling [7] have fallen out of favor in preference for in-home collection kits. These methods require volunteers to sample a small amount of faecal 
material, but standardization of subsampling in the context of the complete bowel movement is rarely stipulated in available protocols. Given consideration of the implications of other aspects of sample collection/storage/ handling on overall microbial composition, it is surprising that the potential impacts of faecal subsampling have been less readily documented. Of the few studies we are aware of, findings are inconsistent; Gorzelak et al. [8] have previously reported differences in the abundance of a number of key taxa in subsamples taken from outer and inner stool microenvironments, whereas Santiago et al. [9] reported largely similar composition from outer and inner stool microenvironments. Conscious of the apparent lack of empirical data supporting consistent microbial composition, or otherwise, from faecal subsamples and the considerable practical implications of such observations, the aim of this study was to compare the microbial composition from faecal subsamples collected from the beginning and end of a single bowel movement.

\section{Main text Methods}

This study involved the analysis of faecal material collected as part of the AussieGut ${ }^{\mathrm{TM}}$ program for the determination of faecal microbial composition. Individuals ( $\mathrm{n}=10$; 6 female, 4 male; age: $24-55$ years) were instructed to collect a small $(<5 \mathrm{~g})$ faecal subsample from the beginning and end of a single bowel movement using provided faecal collection kits. Collection kits included a $70 \mathrm{~mL}$ faecal collection cup with scooped lid (Sarstedt, SA, Australia) and flushable collection paper (Eiken Chemical, Tokyo, Japan). Samples were to be free of water and urine. Faecal samples were returned to the laboratory within $24 \mathrm{~h}$ of collection and stored at $-80^{\circ} \mathrm{C}$ until analysis. Individuals provided written informed consent prior to participation and ethical approval was provided by the Griffith University Human Research Ethics Committee (ref\#: MED/19/15/HREC).

Thawed faecal samples were initially homogenized in phosphate buffered saline, followed by repeated cycles of chemical and mechanical (bead beating) lysis and subsequent DNA extraction using a commercially available kit (Qiagen, Hilden, Germany) as has been previously reported [10]. The V3-V4 region of the microbial 16s rRNA marker gene was amplified using universal primers (F: 5'-CCTACGGGNGGCWGCAG-3'; R: 5'-GAC TACHVGGGTATCTAATCC-3') [11], and polymerase chain reaction products sequenced on an Illumina MiSeq system (Illumina, California, USA) by a commercial provider (Macrogen, Seoul, South Korea). Clustering of sequence reads into operational taxonomic units (OTU) at $97 \%$ identity level was achieved using the Quantitative Insights in Microbial Ecology (QIIME) Suite [12]. The ChimeraSlayer program was used to remove chimaeras from aligned OTU and the FastTreeMP tool generated a phylogenetic tree. Taxonomic identity assignment was performed using a reference-based approach with the NCBI database of $16 \mathrm{~S}$ rRNA gene sequences.

Identified microbial taxa were considered as prevalent (present in greater than $75 \%$ of the samples) or not-prevalent (present in less than $25 \%$ of the samples). A principle components analysis ( $\mathrm{PCoA}$ ) was used to explore the difference in the global microbial composition between the subsamples. The relative abundance of prevalent taxa were compared between subsamples using a paired-sample t-test or a Wilcoxon matched-pair signed rank test. Statistical significance was accepted at $\mathrm{p}<0.05$.

\section{Results}

An average of 27,976 \pm 4955 reads per sample were used for taxa assignment. The total OTU count between subsamples did not differ $(\mathrm{p}=0.32)$, with approximately 240 OTU identified (Table 1). Similarly, the Shannon diversity

Table 1 Diversity metrics and relative abundance data for prevalent bacterial phyla between faecal subsamples. Data are presented as mean \pm SD (median; range)

\begin{tabular}{lccc}
\hline & Sub-sample 1 & Sub-sample 2 & p-value \\
\hline Diversity metrics & & & \\
$\quad$ OTU count & $243 \pm 75(243 ; 140-362)$ & $237 \pm 71(235 ; 135-360)$ & 0.32 \\
$\quad$ Shannon Index & $4.69 \pm 0.69(4.83 ; 3.71-5.50)$ & $4.77 \pm 0.69(4.88 ; 3.70-5.75)$ & 0.29 \\
Phyla (relative abundance) & & & \\
Actinobacteria & $0.41 \pm 0.25(0.41 ; 0.02-0.81)$ & $0.42 \pm 0.32(0.29 ; 0.01-0.98)$ & 0.80 \\
Bacteroidetes & $30.9 \pm 12.1(29.8 ; 15.4-49.0)$ & $29.7 \pm 11.2(28.3 ; 16.8-47.1)$ & 0.51 \\
Firmicutes & $63.2 \pm 11.3(58.8 ; 50.0-81.3)$ & $65.7 \pm 10.5(65.7 ; 52.0-81.2)$ & 0.35 \\
$\quad$ Proteobacteria & $1.78 \pm 1.85(1.03 ; 0.19-6.20)$ & $1.22 \pm 0.80(0.99 ; 0.24-2.62)$ & $0.92^{\mathrm{a}}$ \\
$\quad$ Verrucomicrobia & $3.57 \pm 5.83(1.37 ; 0-18.0)$ & $2.78 \pm 4.14(1.06 ; 0.0-12.6)$ & $0.59^{\mathrm{a}}$ \\
\hline
\end{tabular}

${ }^{a} p$ value from Wilcoxon sign-ranked test 
index was consistent between subsamples $(\mathrm{p}=0.29)$. At the phyla level, five phyla were prevalent; the relative abundance of these key phyla were consistent between the subsamples (Table 1). The Cyanobacteria (detected in a single sample only) and Tenericutes phyla (detected in both samples from two individuals) were considered not prevalent in the sample set and persisted at low relative abundance $(<0.05 \%)$.

At the Family level, 44 unique bacterial families were identified; seven of these families were not prevalent within the sample set (Additional file 1: Table S1). A
PCoA using the prevalent families indicated that the microbial composition was generally similar between the subsamples (Fig. 1A). Further consideration of the prevalent families indicated that 35 of the 37 families had a consistent relative abundance between the subsamples; the relative abundance of the unclassified Bacillales $(\mathrm{p}=0.02)$ and the Eubacteriaceae family $(\mathrm{p}=0.03)$ differed between the subsamples. For the unclassified Bacillales, this was accounted for by the detection of the taxa in five of the participants' subsample 1, but in none of the participants' subsample 2 (Fig. 2A). For the
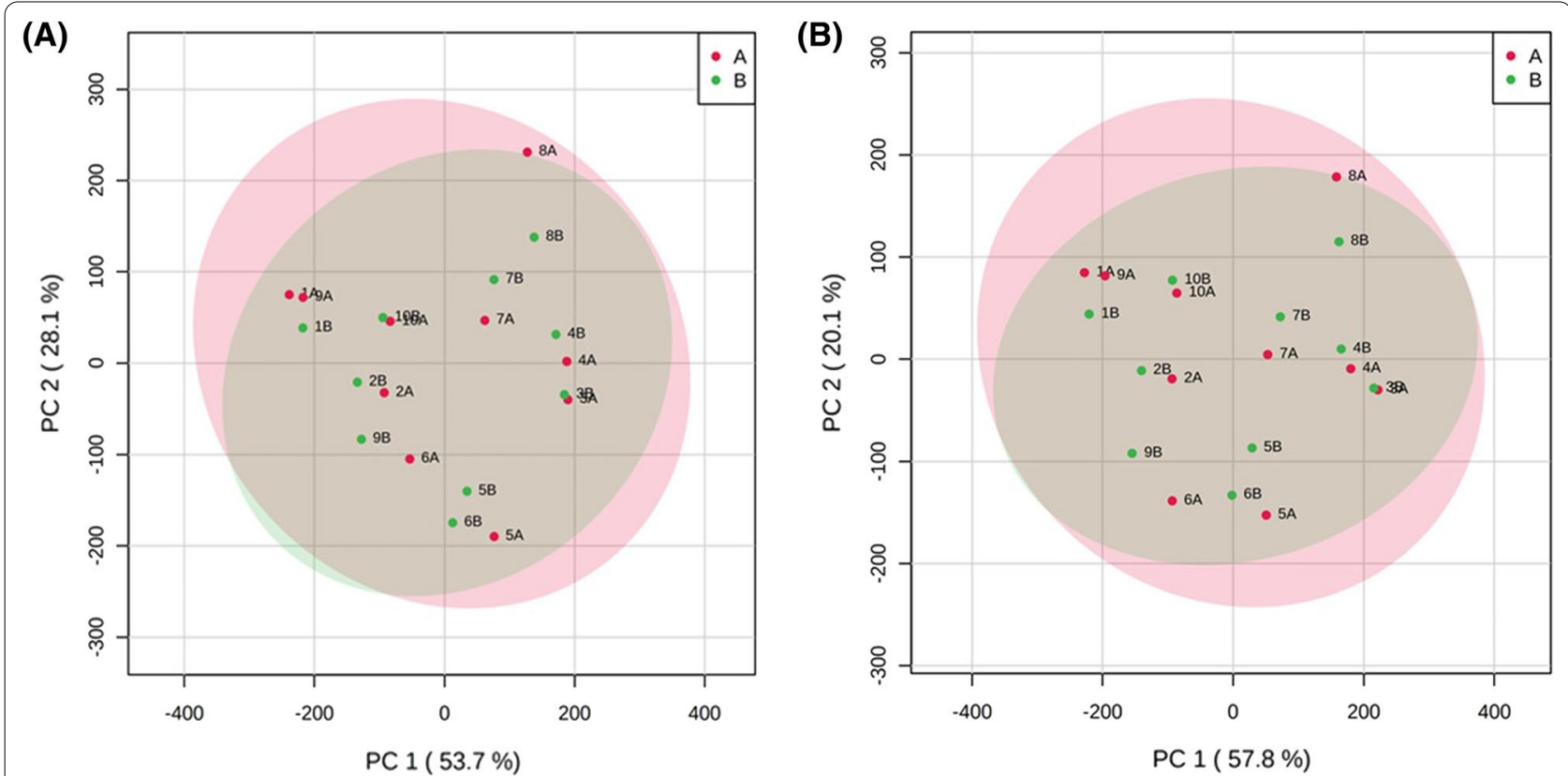

Fig. 1 Principle component analysis for $(\mathbf{A})$ prevalent families (B) prevalent genera
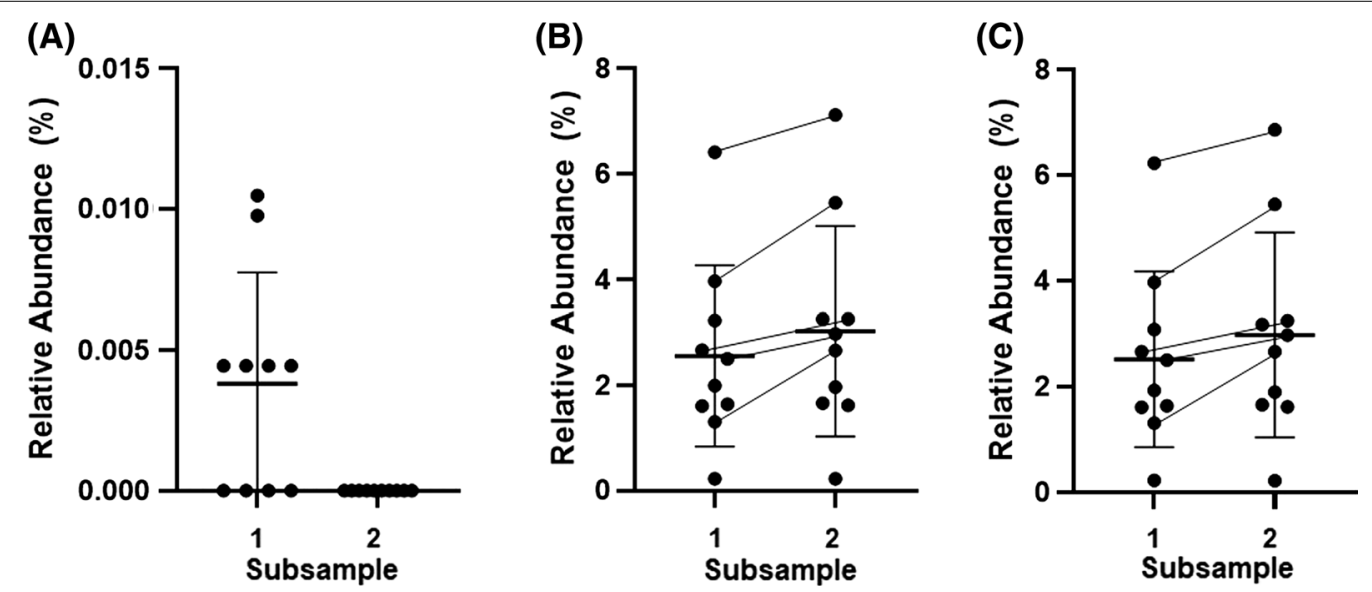

Fig. 2 Relative abundance of (A) unclassified Bacillales (B) Eubacteriaceae family (C) Eubacterium genera between subsample 1 and subsample 2 
Eubacteriaceae family, the difference between the two subsamples was driven by a greater (10\%-twofold) relative abundance in subsample 2 for five participants (Fig. 2B).

At the Genus level, 109 unique bacterial genera were identified; 31 of these genera were not prevalent within the sample set (Additional file 2: Table S2). A PCoA using the prevalent genera indicated that the microbial composition was generally similar between the subsamples (Fig. 1B). Further consideration of the prevalent genera indicated that 77 of the 78 prevalent genera had a consistent relative abundance between the subsamples; the relative abundance of the Eubacterium genera $(p=0.03)$ differed between the subsamples. This difference was driven by a greater ( $10 \%$ - twofold) relative abundance in subsample 2 for five participants (Fig. 2C).

\section{Discussion}

Efforts to better understand the implications of various methodological choices on the study of microbial composition are well documented $[1,2]$. However, the potential for variability in faecal subsamples from a single bowel movement appears less well characterized. With the move to greater use of at-home, low-volume faecal collection kits to facilitate large population-based studies, empirical data to support consistent microbial composition between faecal subsamples from a single bowel movement is crucial. This study was able to demonstrate that global diversity metrics and composition to the genus level were broadly consistent between faecal subsamples collected from the beginning and end of a single bowel movement in otherwise healthy adults.

Quantification of the inherent variability in microbial composition, both within a single bowel movement and within consecutive bowel movements (in the absence of other intervention or dietary modification), appears relatively limited. In contrast, attempts to document spatial heterogeneity in microbial composition along the gastrointestinal tract are more common [13] and suggest differences in composition may persist even between relatively close anatomical sampling sites such as the sigmoid colon and rectum [14]. Our study has been able to help clarify if such differences carry over to luminal contents and create distinct niches within a single bowel movement. Our findings of broadly consistent microbial diversity and composition between faecal subsamples are also in agreement with the prior report from Santiago et al. [9] who found microbial composition was largely similar between outer and inner stool microenvironments. We do make note of the tendency for increased relative abundance of the anaerobic Eubacterium genera, in the subsample collected from the end of the bowel movement among half of the participants, however it is unclear why this trend was evident for this one genera only and other members of the Clostridia class were not similarly impacted.

\section{Limitations}

We acknowledge that the study is not without its limitations, employing a modest sample size and collection of subsamples from the beginning and end of the bowel movement only. Aware of the limitation of $16 \mathrm{~s}$ methodologies for species level identification [15], we chose to report to the genus level only and so it is unclear if the largely consistent compositional profiles between subsamples extend beyond the genus level and include rare taxa. In addition, our study recruited otherwise healthy adults only and it is not clear if findings of consistent compositional features between subsamples would also be observed in particular disease states where skewed microbial composition may be anticipated. However, the outcomes do provide assurance that use of methodologies which employ collection of a faecal subsample can provide representation of the microbial composition from the gut luminal contents more broadly.

\section{Abbreviations}

OTU: Operational taxonomic units; QIIME: Quantitative Insights in Microbial Ecology; PCoA: Principle components analysis.

\section{Supplementary Information}

The online version contains supplementary material available at https://doi. org/10.1186/s13104-022-05923-6.

Additional file 1: Table S1. Prevalence and relative abundance of bacterial families from five key phyla for paired faecal subsamples.

Additional file 2: Table S2. Prevalence and relative abundance of bacterial genera from five key phyla for paired fecal subsamples.

\section{Acknowledgements}

The authors gratefully acknowledge the efforts of the participants in the study.

\section{Authors' contributions}

AJC, AWC and NPW were responsible for study conceptualisation and design. AJC and RR managed recruitment and sample collection. AJC and LH were responsible for the sample analysis. AJC, TMN and KMH performed the data analysis. All authors read and approved the final manuscript.

\section{Funding}

There is no external funding to declare.

Availability of data and materials

The datasets used and/or analysed during the current study are available from the corresponding author on reasonable request.

\section{Declarations}

Ethics approval and consent to participate

Ethical approval was provided by the Griffith University Human Research Ethics Committee (ref\#: MED/19/15/HREC). Individuals provided written informed consent prior to participation. 


\section{Consent for publication}

Not applicable.

\section{Competing interests}

The authors declare that they have no competing interests.

\section{Author details}

${ }^{1}$ School of Medical Science, Griffith Health Centre (G40), Griffith University, Parklands Drive, Southport, QLD 4215, Australia. ${ }^{2}$ Menzies Health Institute Queensland, Griffith University, Southport, QLD, Australia. ${ }^{3}$ Queensland Cyber Infrastructure Foundation Ltd Bioinformatics, St Lucia, QLD, Australia. ${ }^{4}$ School of Medicine, Griffith University, Southport, QLD, Australia.

Received: 14 August 2021 Accepted: 26 January 2022

Published online: 14 February 2022

\section{References}

1. Pollock J, Glendinning L, Wisedchanwet T, Watson M. The madness of microbiome: attempting to find consensus "Best Practice" for $16 \mathrm{~S}$ microbiome studies. Appl Environ Microbiol. 2018;84:e02627-e2717.

2. Vandeputte D, Tito RY, Vanleeuwen R, Falony G, Raes J. Practical considerations for large-scale gut microbiome studies. FEMS Microbiol Rev. 2017:41:S154-67.

3. Tedjo DI, Jonkers DM, Savelkoul PH, Masclee AA, van Best N, Pierik MJ, Penders J. The effect of sampling and storage on the fecal microbiota composition in healthy and diseased subjects. PLoS ONE. 2015;10:e0126685.

4. Videnska P, Smerkova K, Zwinsova B, Popovici V, Micenkova L, Sedlar K, Budinska E. Stool sampling and DNA isolation kits affect DNA quality and bacterial composition following 16S rRNA gene sequencing using MiSeq Illumina platform. Sci Rep. 2019;9:13837.

5. Choo JM, Leong LE, Rogers GB. Sample storage conditions significantly influence faecal microbiome profiles. Sci Rep. 2015:5:16350.

6. Flores R, Shi J, Yu G, Ma B, Ravel J, Goedert JJ, Sinha R. Collection media and delayed freezing effects on microbial composition of human stool. Microbiome. 2015;3:33.

7. Hsieh YH, Peterson CM, Raggio A, Keenan MJ, Martin RJ, Ravussin E, Marco ML. Impact of different fecal processing methods on assessments of bacterial diversity in the human intestine. Front Microbiol. 2016;7:1643.

8. Gorzelak MA, Gill SK, Tasnim N, Ahmadi-Vand Z, Jay M, Gibson DL. Methods for improving human gut microbiome data by reducing variability through sample processing and storage of stool. PLoS ONE. 2015;10:e0134802.

9. Santiago A, Panda S, Mengels G, Martinez X, Azpiroz F, Dore J, Guarner F, Manichanh C. Processing faecal samples: a step forward for standards in microbial community analysis. BMC Microbiol. 2014;14:112.

10. Yu Z, Morrison M. Improved extraction of PCR-quality community DNA from digesta and fecal samples. Biotechniques. 2004;36:808-12.

11. Klindworth A, Pruesse E, Schweer T, Peplies J, Quast C, Horn M, Glockner FO. Evaluation of general 165 ribosomal RNA gene PCR primers for classical and next-generation sequencing-based diversity studies. Nucleic Acids Res. 2013;41:e1.

12. Caporaso JG, Kuczynski J, Stombaugh J, Bittinger K, Bushman FD, Costello EK, Fierer N, Pena AG, Goodrich JK, Gordon Jl, et al. QIIME allows analysis of high-throughput community sequencing data. Nat Methods. 2010;7:335-6.

13. Hillman ET, Lu H, Yao T, Nakatsu CH. Microbial ecology along the gastrointestinal tract. Microbes Environ. 2017;32:300-13.

14. Zhang Z, Geng J, Tang X, Fan H, Xu J, Wen X, Ma ZS, Shi P. Spatial heterogeneity and co-occurrence patterns of human mucosal-associated intestinal microbiota. ISME J. 2014;8:881-93.

15. Johnson JS, Spakowicz DJ, Hong BY, Petersen LM, Demkowicz P, Chen L, Leopold SR, Hanson BM, Agresta HO, Gerstein M, et al. Evaluation of $16 \mathrm{~S}$ rRNA gene sequencing for species and strain-level microbiome analysis. Nat Commun. 2019;10:5029.

\section{Publisher's Note}

Springer Nature remains neutral with regard to jurisdictional claims in published maps and institutional affiliations.
Ready to submit your research? Choose BMC and benefit from:

- fast, convenient online submission

- thorough peer review by experienced researchers in your field

- rapid publication on acceptance

- support for research data, including large and complex data types

- gold Open Access which fosters wider collaboration and increased citations

- maximum visibility for your research: over 100M website views per year

At BMC, research is always in progress.

Learn more biomedcentral.com/submissions 